\title{
Global Properties of Supermanifolds
}

\author{
Jeffrey M. Rabin ${ }^{1, \star}$ and Louis Crane ${ }^{2}$ \\ 1 The Enrico Fermi Institute of the University of Chicago, Chicago, IL 60637, USA \\ 2 Department of Mathematics, University of Chicago, Chicago, IL 60637, USA
}

\begin{abstract}
We construct new examples of supermanifolds, and determine the vector bundle structure of the supermanifolds commonly used in physics. We show that any supermanifold admits a foliation whose leaves are locally tangent to the soul directions in the coordinate charts, and which is one of a nested sequence of foliations. We point out that the existence of these foliations implies restrictions on the possible topologies of supermanifolds. For example, a compact supermanifold with a single even dimension must have vanishing Euler characteristic. We also show that a globally defined superfield on a "nice" compact supermanifold must be constant along the leaves of the foliations. By this mechanism, the global topology of a supermanifold can be used to impose physically interesting constraints on superfields. As an example, we exhibit a supermanifold which has the local geometry of flat superspace but is such that all globally defined superfields are chiral.
\end{abstract}

\section{Introduction}

Superspace techniques have become standard tools for exploring the properties of supersymmetric field theories [1]. Various attitudes can be taken toward the anticommuting $\theta$ coordinates which are introduced in the superspace approach. One common attitude is to regard superspace as merely a formal or heuristic tool for obtaining results which could be more laboriously derived by other, more rigorous, methods. On this view, the anticommuting coordinates are merely formal symbols serving bookkeeping functions: keeping track of the particle content of supermultiplets as well as the differing commutation relations of bosonic and fermionic fields. Functions of the $\theta$ coordinates are understood in the sense of formal power series only. Although supersymmetry transformations can be formally represented as coordinate transformations in superspace, the real

* Enrico Fermi Fellow. Research supported by the NSF: PHY 83-01221, and the Department of Energy: DE AC 02-82-ER-40073 
content of supersymmetry in any specific field theory is in the existence of conserved spinor-vector currents. These currents may be constructed by superspace techniques, but once constructed they are ordinary fields and their conservation may be independently verified using the equations of motion.

In the formal approach described above, it is never necessary to face the delicate questions of what values the $\theta$ coordinates can assume or how a manifold can have coordinates which anticommute. Such a noncommittal stance is adequate for globally supersymmetric theories, but is less than satisfying for supergravity. The equations of supergravity theory look like those of differential geometry, and seem to demand a geometric interpretation, but this is not possible within the above framework because there is no rigorous discussion of how a manifold can be parametrized with both commuting and anticommuting coordinates. There is no notion of a "supermanifold" on which the superspace symbols $x$ and $\theta$ can be local coordinates.

A mathematically rigorous notion of supermanifold which seems appropriate for physical applications such as supergravity has been given by Rogers [2-4] and further developed by Hoyos et al. [5]. In addition to furnishing a mathematical underpinning for the local geometric constructions of supergravity theory, such as curvature and torsion tensors, it also allows one to study global topological features of supermanifolds. In general relativity one is used to the idea that the local geometry of a manifold influences, but does not generally determine, its global topology. Several different topologies may be compatible with a given local metric satisfying the Einstein field equations, and the different topologies can lead to different physics. Similar phenomena should be expected in supergravity, and Rogers' theory of supermanifolds allows one to investigate them.

In this paper we will present some basic topological results on supermanifolds which may be of physical relevance. We begin in Sect. 2 by reviewing the definition of a supermanifold, stressing the analogy between supermanifolds and complex manifolds which will be an important theme in this paper. All examples of supermanifolds in the literature fall into two classes. The first class contains the supermanifolds normally used in physics, which are constructed as vector bundles over an ordinary real "body" manifold which serves as the physical spacetime. These supermanifolds have no "extra" topology, being homotopy equivalent to their bodies. Supermanifolds of the second class are obtained as quotient spaces of flat superspace by discrete groups. These supermanifolds have first homotopy groups isomorphic to the discrete group used in the construction and can be compact. We present two new examples of supermanifolds, one being a slight generalization of the class one type and the other an example of class two with a nonabelian fundamental group as well as torsion in its first homology group. We also investigate the structure of the vector bundles which can arise as bosonic supermanifolds of type one and show that they are direct sums of copies of the tangent bundle of the body manifold.

In Sect. 3 we point out that any supermanifold having $m$ even dimensions admits a foliation of codimension $m$, as well as an infinite set of foliations of higher codimension. Certain topological conditions must be satisfied in order for a manifold to admit such foliations, and therefore we obtain necessary conditions for a real manifold to admit a supermanifold structure. In the case of a compact 
manifold with a single even dimension, for example, the Euler number must vanish, ruling out spheres and projective spaces.

In Sect. 4 we show that the topology of a supermanifold imposes restrictions on the possible globally defined superfields. These restrictions are similar to those on analytic functions on a complex manifold. They arise because once an analytic function is specified in one coordinate chart it has a unique analytic continuation along a given path to any other point. If the analytic continuations to any point along different paths differ, then the given local function has no global extension. The main result here is that, under technical conditions to be discussed, a globally defined superfield on a compact supermanifold must be constant along the leaves of the foliations. As for complex manifolds, there is a corollary showing that a compact supermanifold cannot be embedded (superanalytically) in flat superspace. Another corollary concerns the triviality of Berezin integration on compact fermionic supermanifolds. In Sect. 5 we illustrate a similar phenomenon of "topological imposition of constraints" by constructing another quotient supermanifold of flat superspace. Here only superfields which are chiral can be globally defined on the quotient space.

\section{Definition and Examples of Supermanifolds}

We begin by stressing the similarity between the definitions of complex manifolds and supermanifolds. To define a complex manifold one begins by defining the complex numbers as an extension of the algebra of real numbers and introducing the concept of an analytic function of several complex variables. A complex manifold of dimension $n$ is then defined as a real manifold of dimension $2 n$ which admits the following additional structure. In each coordinate chart it must be possible to regard the $2 n$ real coordinates as the real and imaginary parts of $n$ complex coordinates, and the transition functions of the coordinate atlas must be analytic functions of these complex variables.

For supermanifolds the role of the complex numbers is played by $B_{L}$, the Grassmann algebra with $L$ generators. The generators, denoted $v_{1}, v_{2}, \ldots, v_{L}$, anticommute under multiplication:

$$
v_{i} v_{j}=-v_{j} v_{i} .
$$

Sequences of subscripts will be used to denote products of generators,

$$
v_{i j . . k}=[\sqrt{-1}] v_{i} v_{j} \ldots v_{k}, \quad i<j<\ldots<k .
$$

The factor $\sqrt{-1}$ in the definition is not necessary in the purely mathematical study of supermanifolds. For applications to supersymmetry, however, it is convenient to make $v_{i j . . k}$ real with respect to a complex conjugation which reverses the order of the $v_{i}$ factors in a product. The factor $\sqrt{-1}$ is included whenever necessary for this purpose. For example, $v_{12}=\sqrt{-1} v_{1} v_{2}$ is real, as is $v_{1234}=v_{1} v_{2} v_{3} v_{4}$. A general element of the Grassmann algebra has the form

$$
\begin{aligned}
A & =A_{0}+A_{i} v_{i}+A_{i j} v_{i j}+\ldots, \quad i<j<\ldots<k \\
& \equiv A_{\Gamma} v_{\Gamma},
\end{aligned}
$$


where all coefficients $A_{\Gamma}$ are real numbers. These numbers are called the components of $A$. The element $A$ is called even or odd if the above expansion contains only products of even or odd numbers of generators. The odd elements mutually anticommute while even elements commute with everything. Even elements are denoted by $x$, odd elements by $\theta$, and elements which may be either even or odd by $z$. An even or odd element is specified by $2^{L-1}$ real parameters. The real number part $A_{0}$ of an element $A$ is called its body while the remainder $A-A_{0}$ is the soul, denoted $s(A)$.

The analog of $C^{m}$ is the flat superspace with $m$ even and $n$ odd coordinates, $B_{L}^{m, n}$. This is simply Euclidean space of dimension $(m+n) 2^{L-1}$ with coordinates labeled $x_{\Gamma}^{\mu}$ and $\theta_{\Gamma}^{\alpha}, \mu=1,2, \ldots, m ; \alpha=1,2, \ldots, n$. From these real coordinates one constructs Grassmann coordinates $x^{\mu}$ as in Eq. (2.3).

The analogs of the complex analytic functions are superfields, $B_{L}$-valued functions on flat superspace which have (terminating) Taylor expansions in powers of the $\theta$ coordinates,

$$
F(x, \theta)=f_{0}(x)+f_{\alpha}(x) \theta^{\alpha}+\ldots+f_{12 \ldots n}(x) \theta^{1} \theta^{2} \ldots \theta^{n},
$$

where the functions of $x$ appearing on the right are also required to have (terminating) expansions in powers of the soul coordinates,

$$
f(x)=f\left(x_{0}\right)+s\left(x^{\mu}\right) \partial_{\mu} f\left(x_{0}\right)+\frac{1}{2} s\left(x^{\mu}\right) s\left(x^{\nu}\right) \partial_{\mu} \partial_{v} f\left(x_{0}\right)+\ldots .
$$

If the functions $f_{\alpha \beta \ldots \delta}\left(x_{0}\right)$ are all real analytic, the superfield is called superanalytic. If they are only assumed to be infinitely differentiable, as is usual in physics, we obtain the slightly weaker concept of a $G^{\infty}$ superfield. A superanalytic superfield has a power series expansion about any point in superspace, and the property of unique analytic continuation from one open set into another overlapping one. For $G^{\infty}$ superfields unique analytic continuation is only possible along soul directions.

We can now define a superanalytic or $G^{\infty}$ supermanifold of dimension $(m, n)$ as a real manifold of dimension $(m+n) 2^{L-1}$ such that in each coordinate chart the real coordinates can be grouped into $m x$ and $n \theta$ coordinates in such a way that the transition functions are superanalytic or $G^{\infty}$. It follows trivially that any real manifold of the appropriate dimension which can be covered by a single coordinate chart is automatically a supermanifold; in particular $B_{L}^{m, n}$ is a supermanifold. We will implicitly assume that all supermanifolds considered in this paper are connected.

Relatively few examples of supermanifolds are known; as in the complex case, it is not trivial to determine whether a given real manifold admits a superanalytic structure. One class of known examples consists of the "Grassmann extensions" of real manifolds $[2,6]$. From a given $m$-dimensional "spacetime" manifold one can construct first a canonical $(m, 0)$-dimensional supermanifold, and then an $(m, n)$ supermanifold by taking the direct product with $B_{L}^{0, n}$. This is the structure of superspace assumed in supergravity theories.

Given a smooth $m$-dimensional manifold $M$, with coordinate charts $U_{j}$ and coordinate maps $\phi_{j}: U_{j} \rightarrow R^{m}$, the transition functions are

$$
g_{i j}=\phi_{j} \phi_{i}^{-1}: \phi_{i}\left(U_{i} \cap U_{j}\right) \rightarrow \phi_{j}\left(U_{i} \cap U_{j}\right) .
$$


As coordinate charts in the supermanifold to be constructed, take the regions

$$
S_{j}=\left\{x \text { in } B_{L}^{m, 0} \text { with } x_{0} \text { in } \phi_{j}\left(U_{j}\right)\right\} .
$$

$S_{j}$ is a cylinder over $\phi_{j}\left(U_{j}\right)$. By inserting $g_{i j}$ for $f$ in Eq. (2.5) we extend the domain of definition of $g_{i j}$ to every point of $S_{i}$ whose body lies in $\phi_{i}\left(U_{i} \cap U_{j}\right)$ and these extended transition functions are $G^{\infty}$. The supermanifold of dimension $(m, 0)$ is obtained from the disjoint union of the sets $S_{j}$ by identifying each point of $S_{i}$ which is in the domain of $g_{i j}$ with its image in $S_{j}$. (This is a standard method of constructing manifolds with prescribed transition functions.) The resulting supermanifold is a vector bundle over the real manifold from which the construction began. The real manifold is called the body of the supermanifold.

It is of interest to determine the structure of these Grassmann extensions as vector bundles. This can be done by examining the transition functions. To avoid proliferation of indices, consider the overlap of two specific charts on the $(m, 0)$ supermanifold, with coordinates $x^{\mu}$ and $y^{\mu}$, and let the transition functions be $y^{\mu}$ $=f^{\mu}(x)$, where $f^{\mu}$ is $G^{\infty}$ as in Eq. (2.5). By equating the coefficients of $v_{\Gamma}$ on both sides of this equation, we obtain the transition functions in terms of the real coordinates:

$$
\begin{aligned}
y_{0}^{\mu} & =f^{\mu}\left(x_{0}\right), \\
y_{i j}^{\mu} & =\partial_{v} f^{\mu}\left(x_{0}\right) x_{i j}^{\nu}, \\
y_{i j k l}^{\mu} & =\partial_{v} f^{\mu}\left(x_{0}\right) x_{i j k l}^{\nu}+\partial_{v} \partial_{\lambda} f^{\mu}\left(x_{0}\right)\left(x_{i j}^{\nu} x_{k l}^{\lambda}-x_{i k}^{\nu} x_{j l}^{\lambda}+x_{i l}^{\nu} x_{j k}^{\lambda}\right),
\end{aligned}
$$

and so forth.

The first equation simply states that $f^{\mu}\left(x_{0}\right)$ are the transition functions on the body. The second says that for each set of values $i j, x_{i j}^{\mu}$ transforms by the Jacobian matrix of the transition functions of the body. Hence this set of variables may be taken as fiber coordinates in many copies of the tangent bundle of the body. However, the interpretation of the remaining equations is not obvious. The general form of the equations is that a coordinate $y_{\Gamma}^{\mu}$ depends linearly on coordinates with the same number of subscripts, and polynomially on coordinates with fewer subscripts. Strictly speaking, this means that the supermanifold is actually not a vector bundle, which by definition has linear transition functions, but is merely a fiber bundle whose fiber is a vector space. However, we can find an equivalent bundle whose transition functions are linear. The point is that the coordinates which make manifest the $G^{\infty}$ structure differ from those which expose the linear structure.

We rewrite the transition functions schematically as

$$
\begin{gathered}
y_{(L)}=A x_{(L)}+P\left(x_{(L-2)}, x_{(L-4)}, \ldots\right), \\
y_{(L-2)}=B x_{(L-2)}+Q\left(x_{(L-4)}, x_{(L-6)}, \ldots\right),
\end{gathered}
$$

and so forth. We have assumed for definiteness that $L$ is even. Here $x_{(k)}$ is the column vector of coordinates with $k$ subscripts, $A, B, \ldots$ are matrices of partials of $f^{\mu}$, and $P, Q, \ldots$ are polynomials. We can continuously deform the transition functions by letting $P \rightarrow t P$ and shrinking $t$ from 1 to 0 . One can check that this prescription can be consistently applied in all coordinate patches and that the 
transition functions remain invertible for all $t$. The next step is to replace $Q \rightarrow s Q$ and shrink $s$, and in this way we successively remove the polynomial terms from Eqs. (2.7). There are various ways to see that such a deformation of transition functions results in an equivalent bundle [7]. The final conclusion is that the $(m, 0)$ Grassmann extension of a real manifold is equivalent as a bundle to the direct sum of $2^{L-1}-1$ copies of the tangent bundle of the body.

As noted above, fermionic coordinates are normally included simply by taking the direct product of the $(m, 0)$ Grassmann extension with $B_{L}^{0, n}$. We would like to point out that any rank $n$ vector bundle over the body can be used to construct the fermionic sector. Let the typical transition function of such a rank $n$ bundle be $u^{\prime \alpha}$ $=M_{\alpha \beta}\left(x_{0}\right) u^{\beta}$, where $u^{\alpha}$ are the fiber coordinates and $M_{\alpha \beta}$ is a matrix of functions of $x_{0}$. We extend these functions to $G^{\infty}$ functions of $x$ using Eq. (2.5) and construct the fermionic sector as the bundle over the $(m, 0)$ Grassmann extension whose transition functions are $\theta^{\prime \alpha}=M_{\alpha \beta}(x) \theta^{\beta}$. A homotopy argument like that above shows that this supermanifold has the structure of the direct sum of $2^{L-1}-1$ copies of the tangent bundle to the body and $2^{L-1}$ copies of the chosen rank $n$ vector bundle.

Many supermanifolds which are not actually defined as Grassmann extensions of real manifolds nevertheless turn out to be of this type. We we would like to present a new example of such a supermanifold, namely super projective space, denoted $S P_{L}^{m, n}$. The definition is analogous to that of complex projective space. Begin with the flat superspace $B_{L}^{m+1, n}$ and remove the points $(x, \theta)$ such that all $x_{0}^{\mu}=0$. Take the multiplicative group whose elements $g$ are the even, invertible elements of $B_{L}$ and let it act on the remaining points by

$$
g\left(x^{\mu}, \theta^{\alpha}\right)=\left(g x^{\mu}, g \theta^{\alpha}\right) .
$$

The space $S P_{L}^{m, n}$ will be the quotient space obtained by identifying all points related by group transformations.

We show that $S P_{L}^{m, n}$ is a supermanifold by explicitly constructing a coordinate atlas. There will be $m+1$ charts $S_{j}$, where $S_{j}$ is the set of equivalence classes of points $\left(x^{\mu}, \theta^{\alpha}\right)$ having $x_{0}^{j} \neq 0$, so that $x^{j}$ is invertible. Any equivalence class in $S_{j}$ contains a unique point with $x^{j}=1$, and we take its coordinates $\left(x^{1}\right.$, $\left.x^{2}, \ldots, 1, \ldots, x^{m+1}, \theta^{\alpha}\right)$ as the coordinates of this equivalence class in the chart $S_{j}$. It is convenient to retain the superfluous constant coordinate 1 in the notation. If a point lies in $S_{i} \cap S_{j}$ and its coordinates in the chart $S_{i}$ are $\left(x^{\mu}, \theta^{\alpha}\right)$, then its coordinates in the chart $S_{j}$ are $\left[x^{\mu}\left(x^{j}\right)^{-1}, \theta^{\alpha}\left(x^{j}\right)^{-1}\right]$. Therefore the transition functions are superanalytic.

From the structure of the coordinate atlas of $S P_{L}^{m, 0}$ it can be seen that this supermanifold is exactly the Grassmann extension of $m$-dimensional real projective space. Examination of the transition functions also shows that the fermionic sector of $S P_{L}^{m, n}$ is constructed as described above using the rank $n$ vector bundle which is the direct sum of $n$ copies of the canonical line bundle over $R P^{m}$. (Recall that a point of $R P^{m}$ represents a line through the origin in $R^{m+1}$; the canonical line bundle simply takes this line as the fiber over this point.) One may now ask whether $S P_{L}^{m, n}$ is equivalent to $S P_{L}^{m, 0} \times B_{L}^{0, n}$. This depends on whether the appropriate direct sum of line bundles is trivial, a rather difficult mathematical question whose 
answer we quote from [8]. Let $k$ denote the number of integers from 1 to $m$ inclusive which are congruent to $0,1,2$, or $4 \bmod 8$. Then $S P_{L}^{m, n}$ is equivalent to $S P_{L}^{m, 0} \times B_{L}^{0, n}$ iff $2^{k}$ divides $2^{L-1} n$. In particular, this is satisfied if $L>m$ as is the case in any physical application. However, it does show that $S P_{L}^{m, n}$ is not always a supermanifold of the simplest Grassmann extension type.

Quotient spaces of supermanifolds by group actions form a potentially rich source of examples of supermanifolds. If the group acts freely and properly, then the quotient space is a manifold [9]. If in addition the action is $G^{\infty}$, in the sense that the transformed coordinates of any point are $G^{\infty}$ functions of the old coordinates, then the quotient manifold is a supermanifold. Some known examples of this type arise as quotients of flat superspace by discrete group actions. For example, the quotient space of $B_{2}^{1,0}$ by the group generated by unit translations along the $x_{0}$ and $x_{12}$ axes is a torus, which is therefore a supermanifold [2]; this generalizes immediately to higher-dimensional tori. This example also illustrates that a given manifold may admit more than one superanalytic structure. The quotient of $B_{2}^{1,0}$ by the group generated by unit translations along two orthogonal axes which, however, have irrational slope relative to the $x_{0}$ and $x_{12}$ axes is again the torus; but now the $x_{\Gamma}$ axes project onto the torus as irrational, dense torus knots [5]. These superanalytic structures on the torus are good sources of counterexamples to conjectures about supermanifolds.

When a supermanifold is given as the quotient of $B_{L}^{m, n}$ by the properly discontinuous action of a discrete group $G$, the topology of the supermanifold is completely known: its first homotopy group is $G$ and higher homotopy groups vanish [10]. All known compact supermanifolds seem to be of this type. As a new example, we point out the quotient of $B_{L}^{4,4}$ by the discrete supersymmetry group of Kostelecký and Rabin [11]. The discrete supersymmetry (DSUSY) group is generated by finite translations $T$ and supertranslations $Q$ which act as follows:

$$
\begin{gathered}
T\left(a^{\mu}\right)\left(x^{\mu}, \theta^{\alpha}\right)=\left(x^{\mu}+a^{\mu}, \theta^{\alpha}\right), \\
Q\left(\eta^{\alpha}\right)\left(x^{\mu}, \theta^{\alpha}\right)=\left(x^{\mu}-i \bar{\eta} \gamma^{\mu} \theta, \theta^{\alpha}+\eta^{\alpha}\right) .
\end{gathered}
$$

Here $\gamma^{\mu}$ are the Dirac matrices in the Majorana representation, and the group parameters $a^{\mu}$ and $\eta^{\alpha}$ are even and odd elements of $B_{L}$, respectively, whose components $a_{\Gamma}^{\mu}$ and $\eta_{\Gamma}^{\alpha}$ are restricted to integer values. Some relations obeyed by these generators are

$$
\begin{gathered}
Q(\eta) T(a)=T(a) Q(\eta), \\
Q^{-1}(\eta) Q^{-1}(\varepsilon) Q(\eta) Q(\varepsilon)=T(2 i \bar{\varepsilon} \gamma \eta) .
\end{gathered}
$$

This last relation is the exponentiated version of the fundamental SUSY anticommutator.

The quotient of flat superspace by the DSUSY group is a trivial bundle over its body, a torus [the quotient of $R^{4}$ by the central transformations $T\left(a_{0}^{\mu}\right)$ ], but the fibers are compact and topologically nontrivial. According to Eq. (2.11), the fundamental group is nonabelian, and the commutator subgroup consists of precisely the elements of the form $T(2 i \bar{\varepsilon} \gamma \eta)$. The topological interpretation of this situation is that the closed loops represented by elements of the commutator subgroup are noncontractible but do bound embedded 2-surfaces. The 2-surfaces 
which they bound must themselves be topologically nontrivial since if such a loop bounded a disk then it could be contracted to a point within the disk. Loops not in the commutator subgroup are noncontractible because they do not bound at all. Thus, 2-torsion is present because the loops $T(i \bar{\varepsilon} \gamma \eta)$ do not bound at all, while their doubles $T(2 i \bar{\varepsilon} \gamma \eta)$ do. Such loops are topologically like the central circle on a Möbius band. In the Appendix we discuss a 3-manifold which models the topology of this quotient space but is easier to visualize. This model is similar to Rogers' example of a supermanifold with nonabelian fundamental group [3].

\section{The Foliation Structure}

In this section we discuss the rich structure of foliations which exists on any supermanifold. The existence of these foliations is the most important difference between supermanifolds and complex manifolds. It can be traced directly to algebraic differences between the Grassmann numbers and the complex numbers, specifically the existence of nontrivial ideals in the Grassmann algebra.

In each coordinate chart of a given supermanifold, consider the collection of surfaces on which all the body coordinates $x_{0}^{\mu}$ are constant, while the other coordinates vary freely. If these surfaces join together smoothly when coordinate charts overlap, then we have a foliation: a smooth slicing of the entire supermanifold into submanifolds, or leaves, such that each point lies on a unique leaf. Note that a foliation is a weaker concept than a fibration: it is not claimed that all the leaves are isomorphic, nor that the supermanifold is locally a product of some base manifold with the leaves.

That the leaves do join smoothly when two charts overlap is a consequence of the transition functions being $G^{\infty}$. Let the coordinates in the two charts be $(x, \theta)$ and $(y, \phi)$. Then in the overlap region we have

$$
\begin{gathered}
y^{\mu}=y_{\Gamma}^{\mu} v_{\Gamma}=f_{0}^{\mu}(x)+f_{\alpha}^{\mu}(x) \theta^{\alpha}+f_{\alpha \beta}^{\mu}(x) \theta^{\alpha} \theta^{\beta}+\ldots, \\
f_{0}^{\mu}(x)=f_{0}^{\mu}\left(x_{0}\right)+s\left(x^{\nu}\right) \partial_{v} f_{0}^{\mu}\left(x_{0}\right)+\ldots
\end{gathered}
$$

Because the body coordinate $y_{0}^{\mu}$ is by definition that part of $y^{\mu}$ which contains no factors $v_{j}$, only the first term on the right sides of each of Eqs. (3.1) and (3.2) can contribute to it. All other terms explicitly contain factors $v_{j}$. Thus the body coordinates of one chart depend only on the body coordinates of the other chart. This means that in the overlap region, a leaf of constant $x_{0}^{\mu}$ must lie entirely within a leaf of constant $y_{0}^{\mu}$, and vice versa. Therefore the leaves coincide in the overlap regions and we have a foliation, which we will call the soul foliation. For a supermanifold of dimension $(m, n)$, the leaves of the soul foliation have codimension $m$.

The existence of this foliation is implicit but not spelled out in the discussion of the existence of bodies of supermanifolds in [5]. Roughly speaking, if this foliation were a fibration, then the body of a supermanifold could be defined as the base space. For those supermanifolds which are bundles over their bodies, the leaves of the soul foliation are precisely the fibers. The irrational superanalytic structure on 
the torus provides an example in which we do not have a fibration: the leaves are the dense torus knots which correspond to lines parallel to the $x_{12}$ axis in the covering space $B_{2}^{1,0}$.

The existence of the soul foliation is a direct consequence of the existence of an ideal in the Grassmann algebra $B_{L}$. This ideal is the set of elements $A$ such that $A_{0}=0$; this set is closed under multiplication by arbitrary elements of $B_{L}$, and under addition of its own elements. This in turn is true because the Grassmann algebra does not contain inverses for the generators $v_{j}$.

The existence of additional foliations can be deduced from Eqs. (3.1), (3.2), and the analogous equations for the $\phi$ coordinates. For example, the coordinate $y_{i j}^{\mu}$ of one chart can depend only on the coordinates $x_{i j}^{\nu}, \theta_{i}^{\alpha}, \theta_{j}^{\alpha}$, and $x_{0}^{\nu}$ of the other chart. This is because it only gets contributions from terms containing $v_{i}, v_{j}$, and no other generators. In general, a coordinate $y_{\Gamma}^{\mu}$ can depend only on coordinates whose subscripts form a subsequence of $\Gamma$ ( 0 is considered a subsequence of anything). Therefore, for each fixed $i$ and $j$, the surfaces of constant $x_{i j}^{\mu}, \theta_{i}^{\alpha}, \theta_{j}^{\alpha}$, and $x_{0}^{\mu}$ in all charts will piece together to give a foliation.

The ideal of $B_{L}$ corresponding to the foliation just described is the set of elements $A$ with $A_{\Gamma}=0, \Gamma=0, i, j, i j$. A supermanifold will have a foliation corresponding to each ideal of the Grassmann algebra. In each chart the foliation will consist of the surfaces of points whose coordinates differ by elements of the ideal. When the ideal consists of multiples of one of the $v_{\Gamma}$, this can be seen from Eqs. (3.1) and (3.2). More generally it holds because a $G^{\infty}$ function $F(z+\delta z)$ always has a Taylor expansion when $\delta z_{0}=0$. If $\delta z$ belongs to an ideal, so will $F(z+\delta z)$ $-F(z)$. Therefore the defining property of the foliation is preserved under $G^{\infty}$ changes of coordinates.

Thus, a supermanifold has an extremely rich foliation structure which mirrors the algebraic structure of $B_{L}$. The leaves of each foliation are contained in those of the soul foliation, but thereafter the relations of inclusion and intersection among the leaves become complicated. We feel that such a rich structure must impose such strong constraints on possible supermanifolds as to permit a complete classification, but so far our results in this direction are rather modest.

The existence of even a single foliation is a special property for a manifold, and certain topological conditions must be met for it to be possible. The simplest condition applies to a compact manifold $M$ with a foliation of codimension 1, such as the soul foliation on a $(1, n)$ supermanifold. If $M$ is orientable, and if the leaves of the foliation can be given smoothly varying orientations compatible with that of $M$, then there is a field of unit normal vectors to the leaves which is also a unit tangent vector field on $M$. It is well known that the necessary condition for a unit tangent vector field is the vanishing of the Euler number $\chi$ [12].

If the orientability conditions are not met, there is still a field of normal lines to the leaves, tangent to $M$, but a smooth choice of directions on these lines may not be possible. One can then deal with a double cover of $M$ constructed as follows. Choose a base point $P_{0}$ and one of the two normals to the leaf through $P_{0}$ at $P_{0}$. Consider the space of pairs $(P, C)$ where $P$ is a point of $M$ and $C$ is a curve from $P_{0}$ to $P$. A smooth choice of normals to the leaves encountered along $C$ will produce one of the two possible normals at $P$; identify all pairs $(P, C)$ and $(P, C)$ which give the same normal at $P$. The resulting space is a double cover of $M$ which admits a 
nonvanishing tangent vector field and therefore has $\chi=0$. The Euler number of $M$ is half that of its double cover and thus also vanishes.

The vanishing of the Euler number is thus necessary (but not sufficient) for a compact manifold to be a $(1, n)$ supermanifold. Among compact 2-manifolds, for example, only the torus and the Klein bottle can be $(1,0)$ supermanifolds over $B_{2}$. Similarly, no simply connected compact 4-manifold can qualify because Poincaré duality shows that $\chi=2+b_{2}$, where $b_{2}$ is the second Betti number. In higher dimensions, spheres are ruled out ( $\chi=2$ for even-dimensional spheres), as are real $(\chi=1)$ and complex $(\chi=d+1)$ projective spaces of even dimension $2 d$. (Of course, all nontrivial supermanifolds are even-dimensional.)

It is worth pointing out that all Lie groups have $\chi=0$, so that we get no constraints on which Lie groups can be $(1 ; n)$ supergroups.

Relatively little is known about the topological restrictions on foliations of codimension greater than one. It is known, for example, that for $k>3, C P^{k}$ does not admit foliations of codimension 2 , so that it cannot be a $(2, n)$ supermanifold [13], but we are not aware of similar general results for higher codimensions or other manifolds.

\section{Superfields on Compact Supermanifolds}

In this section we will derive the constraint on superfields which is analogous to the fact that an analytic function on a compact connected complex manifold must be constant. Our result is that, under technical assumptions to be discussed, a superfield on a compact supermanifold must be constant along the leaves of the soul foliation. This fact is illustrated in an example and proven for a restricted class of supermanifolds in ref. 5. Because the arguments in this section are more technical than those elsewhere in this paper, we will try to explain the intuition behind the results as well as giving formal proofs. The basic intuition behind the result is that according to Eqs. (2.4) and (2.5), a superfield is a polynomial function of all soul coordinates. However, a smooth real-valued function on a compact manifold must be bounded, and the only bounded polynomials are constants. The two obstructions to extending this intuition to a proof are the facts that superfields are not real-valued and that Eqs. (2.4) and (2.5) hold only in a coordinate chart and not globally.

Theorem 1. A superfield on a compact $(0, n)$ supermanifold is constant.

Proof. Let the globally defined superfield be $f$ and write $f=f_{\Gamma} v_{\Gamma}$. Construct the globally defined smooth real-valued function

$$
\|f\|^{2} \equiv \sum_{\Gamma}\left(f_{\Gamma}\right)^{2}
$$

We have now overcome the first obstruction, and $\|f\|^{2}$ must attain its maximum value at some point $P$ of the supermanifold. Find a chart containing $P$ and choose coordinates so that $P$ is the origin. The remainder of the proof is a local argument within this single chart which shows that the origin cannot be a maximum but at best a saddle point, if $f$ is nonconstant. 
To understand the idea of the argument, consider first the explicit case of a $(0,2)$ supermanifold over $B_{2}$. Then there are four coordinates $\theta_{i}^{\alpha}$, and the most general form of a superfield is

$$
f(\theta)=a+b \theta^{1}+c \theta^{2}+d \theta^{1} \theta^{2},
$$

from which we find

$$
\begin{aligned}
\|f\|^{2}= & a_{0}^{2}+\left(a_{1}+b_{0} \theta_{1}^{1}+c_{0} \theta_{1}^{2}\right)^{2}+\left(a_{2}+b_{0} \theta_{2}^{1}+c_{0} \theta_{2}^{2}\right)^{2} \\
& +\left(a_{12}+b_{1} \theta_{2}^{1}-b_{2} \theta_{1}^{1}+c_{1} \theta_{2}^{2}-c_{2} \theta_{1}^{2}+d_{0} \theta_{1}^{1} \theta_{2}^{2}-d_{0} \theta_{2}^{1} \theta_{1}^{2}\right)^{2} .
\end{aligned}
$$

The general form of a term here is $(a+p)^{2}=a^{2}+p^{2}+2 a p$, with $p$ a polynomial in the $\theta_{i}^{\alpha}$ which vanishes at the origin. The terms $a^{2}+p^{2}$ are clearly nondecreasing in all directions leaving the origin, so the origin can only be a maximum if the sum of all cross terms $2 a p$ alone has a maximum there. This requires first that the cross terms linear in $\theta$ vanish. Then, the quadratic cross terms are $2 a_{12} d_{0}\left(\theta_{1}^{1} \theta_{2}^{2}-\theta_{2}^{1} \theta_{1}^{2}\right)$. But this expression has a saddle point at the origin because of the antisymmetry in the subscripts. To see this, suppose the expression is decreasing leaving the origin in the direction of some vector

$$
V=\sum_{\alpha, i} \hat{\theta}_{i}^{\alpha} .
$$

Then by interchanging subscripts 1 and 2 in $V$, we produce a direction in which the expression must increase. The final conclusion is that the origin can only be a maximum if all the cross terms vanish, whereupon the remaining expression clearly has a minimum at the origin unless it is constant. Constancy of $\|f\|^{2}$ implies constancy of $f$.

The general case of a $(0, n)$ supermanifold over $B_{L}$ is similar. The issue rests on the behavior of the cross terms in the expression for $\|f\|^{2}$. We successively prove the vanishing of the cross terms which are linear, quadratic, cubic, etc. There certainly cannot be an extremum at the origin if the lowest-order cross terms have odd degree, and terms of even degree are handled by an antisymmetry argument like that above. To prove the vanishing of quadratic cross terms, for example, choose two specific values for $\alpha$ and two specific values for $\Gamma$ and restrict the expression to the four-dimensional subspace of these coordinates $\theta_{\Gamma}^{\alpha}$. Antisymmetry in the subscripts then shows that the origin is a saddle point in this subspace, as above, and running through all possible choices of $\alpha$ and $\Gamma$ completes the proof in general.

Corollary. Consider a supermanifold which is the Cartesian product of an $(m, 0)$ supermanifold with a compact $(0, n)$ supermanifold. The Berezin integral of any superfield on this supermanifold is zero.

Strictly speaking, of course, there is no definition of Berezin integration on an arbitrary supermanifold. However, one presumably wants to retain the property that the integral of a constant should be zero, and the result follows.

In order to prove similar results for supermanifolds with even coordinates, it is necessary to make a technical assumption. 
Definition. We say that a supermanifold is nicely foliated, or simply nice, if the $G^{\infty}$ atlas is such that each leaf of the soul foliation intersects each chart at only finitely many values of the body coordinates $x_{0}$.

Theorem 2. A superfield on a nice compact $(m, 0)$ supermanifold must be constant along each leaf of the soul foliation. Equivalently, in each chart it depends on the body coordinates $x_{0}$ only.

The previous theorem rested on the fact that the squared norm of a $G^{\infty}$ function of odd coordinates could not have even local extrema. Unfortunately, this is not true for functions of even coordinates. For example, consider the function on $B_{2}^{1,0}$,

$$
f(x)=1-x^{2}=1-x_{0}^{2}-2 x_{0} x_{12} v_{12} \text {. }
$$

Then $\|f\|^{2}=\left(1-x_{0}^{2}\right)^{2}+\left(2 x_{0} x_{12}\right)^{2}$ does have a local maximum at the origin, though not a global one. This means that the proof of the present theorem will have to be more global in character than that of Theorem 1 .

To clarify the theorem, examine Eq. (2.5), which we repeat here.

$$
f(x)=f\left(x_{0}\right)+s\left(x^{\mu}\right) \partial_{\mu} f\left(x_{0}\right)+\frac{1}{2} s\left(x^{\mu}\right) s\left(x^{\nu}\right) \partial_{\mu} \partial_{v} f\left(x_{0}\right)+\ldots .
$$

Note that the dependence of a superfield on the soul coordinates is determined by the derivatives of its restriction to the body. There are only two ways for a superfield to be independent of the soul coordinates: either $f\left(x_{0}\right)$ must be constant, or it must be of order at least $L-1$ in the generators of the algebra so that multiplication by any two additional generators annihilates it. As an example of the latter possibility, form the torus from $B_{2}^{1,0}$ by using unit translations in the $x_{0}$ and $x_{12}$ directions, and observe that $f(x)=v_{12} \sin 2 \pi x$ is a global superanalytic function.

For the proof, we will first replace the global superfield by an auxiliary field which is linear in the soul coordinates rather than polynomial. Assuming that this field is not constant along the leaves of the soul foliation, we will find a path within one leaf along which some component of the field increases without bound, contradicting the compactness. The technical part of the theorem is to establish a lower bound on the rate of increase of this component per unit path length, and to show that the path can be prolonged indefinitely.

Proof of Theorem 2. Again let $f$ be the global superfield and write $f=f_{\Gamma} v_{\Gamma}$. The terms in this sum containing the smallest number of generators $v_{j}$ will be called the leading terms of $f$. If the leading terms are constant we can globally subtract them off and obtain new leading terms; thus we can assume that the leading terms are nonconstant. In any particular coordinate chart, it is clear from Eq. (4.5) that the leading terms of $f$ are the leading terms of $f\left(x_{0}\right)$. Thus, the leading terms are automatically constant along leaves of the soul foliation. Our auxiliary field $F$ will consist of the leading terms of $f$, plus the terms containing two more generators than the leading terms. In a particular chart,

$$
F(x)=G\left(x_{0}\right)+x_{i j}^{\mu} v_{i j} \partial_{\mu} G\left(x_{0}\right)+H\left(x_{0}\right) .
$$

Here $G$ denotes the leading terms of $f\left(x_{0}\right)$, while $H$ consists of the terms in $f\left(x_{0}\right)$ containing two more generators than the leading terms. $F(x)$ is explicitly linear in the soul coordinates. Our goal is to show that $F(x)$ is constant along leaves. This 
will establish that the leading terms of $f$ contain at least $L-1$ generators, as claimed. So we assume the contrary, that the leading terms contain fewer than $L-1$ generators.

Now, because the supermanifold is compact we may assume that the coordinate atlas contains only finitely many charts. Pick some particular chart in which $G\left(x_{0}\right)$ is nonconstant, choose a value of $x_{0}$ at which the partials $\partial_{\mu} G\left(x_{0}\right)$ are not all zero, and consider the leaf through this value of $x_{0}$. We claim that whenever this leaf meets any chart, some partial derivative of the function $G$ in that chart is nonzero on the leaf. This is proved by induction: nonzero partials of $G$ in the initial chart imply that the leading terms were varying from one leaf to the next in that chart. This continues to be true in the overlaps with neighboring charts, hence in the neighboring charts there must also be nonzero partials. An alternative argument is that the transition functions cannot be invertible if the $x_{i j}$ coordinates of one chart do not depend on the $x_{i j}$ coordinates of an overlapping chart. Therefore a function which depends on these coordinates in one chart must also depend on them in overlapping charts. Since the number of charts is finite, and by niceness the leaf meets each chart finitely many times, we can repeat the argument until all intersections with all charts are accounted for. And since the list of values of all the derivatives at all intersections with charts is finite, there is actually a finite lower bound $\delta$ on the magnitudes of the nonzero components of the derivatives in all charts.

We are going to construct a path within the leaf described above along which some component of $F$ increases without bound. One further technical point is needed to guarantee that this path can be extended indefinitely. This is the fact that for a given compact manifold with a given finite cover of coordinate charts, there exists $\varepsilon>0$ such that the ball of radius $\varepsilon$ around any point lies entirely within some one chart of the cover. This is true whether $\varepsilon$ is distance according to some global metric or is coordinate distance in the charts.

To complete the proof, choose an initial point $P$ on the leaf in some particular coordinate chart. If $G$ contains fewer than $L-1$ generators, there will be some soul coordinate $x_{i j}^{\mu}$ on which $F(x)$ depends linearly with slope at least $\delta$. Leaving $P$ along a path for which $x_{i j}^{\mu}$ either increases or decreases as appropriate, with all other coordinates held constant, must increase some component $F_{\Gamma i j}$ at a rate which is not less than $\delta$. Continue until the path enters a new chart, then stop and select a coordinate $x_{i j}^{v}$ on which $F(x)$ depends linearly in the new chart, then continue as above indefinitely. Since the coordinate length of each such path segment can be chosen to be at least $\varepsilon$, the component $F_{\Gamma i j}$ increases by at least $\varepsilon \delta$ at each step. Thus $F_{\Gamma i j}$ is not bounded, and we obtain a contradiction.

The assumption that the supermanifold is nice is not made in order to rule out cases like the irrationally foliated torus in which each leaf is dense. In fact the theorem is even easier to prove, and the conclusion is stronger, in such a case. Since the leading terms of a superfield are constant along leaves, if the leaves are dense then the leading terms are actually globally constant. When this global constant is subtracted off, the same argument applies to the new leading terms. Hence the superfield itself must be globally constant, not merely constant along leaves.

The situation which is avoided by assuming a nice foliation is that in which a leaf meets a chart at infinitely many values $x_{0}$ which accumulate at some value $\tilde{x}_{0}$. 
Then there can be global superfields whose components achieve their maxima on the leaf through $\tilde{x}_{0}$. The proof of Theorem 2 fails in such a case because, while some component of $F$ must increase along the constructed path, the rate of increase may asymptotically approach zero, and $F$ may remain bounded. We do not know examples of such supermanifolds, nor can we prove that there are none.

Theorem 3. A superfield on a nice compact $(m, n)$ supermanifold must be constant along each leaf of the soul foliation.

Proof. This is proved by combining the techniques used in the previous theorems. In local coordinates, the leading terms of the superfield $f$ will take the form

$$
G_{0}\left(x_{0}\right)+G_{\alpha}\left(x_{0}\right) \theta_{i}^{\alpha} v_{i}+G_{\alpha \beta}\left(x_{0}\right) \theta_{i}^{\alpha} \theta_{j}^{\beta} v_{i j}+\ldots .
$$

The leading terms are no longer constant along leaves of the soul foliation, because of the $\theta$ dependence, but they are constant along leaves of the secondary foliation defined by holding all $x_{0}$ and $\theta_{i}$ fixed. Terms containing two more generators than the leading terms can involve dependence on either the $x_{i j}$ or the $\theta_{i j k}$ coordinates. Therefore the auxiliary field $F$ will take the form

$$
\begin{aligned}
F(x)= & G_{0}\left(x_{0}\right)+G_{\alpha}\left(x_{0}\right) \theta_{i}^{\alpha} v_{i}+G_{\alpha \beta}\left(x_{0}\right) \theta_{i}^{\alpha} \theta_{j}^{\beta} v_{i j}+\ldots \\
& +H_{0}\left(x_{0}\right)+H_{\alpha}\left(x_{0}\right) \theta_{i}^{\alpha} v_{i}+H_{\alpha \beta}\left(x_{0}\right) \theta_{i}^{\alpha} \theta_{j}^{\beta} v_{i j}+\ldots \\
& +x_{i j}^{\mu} v_{i j} \partial_{\mu} G_{0}\left(x_{0}\right)+x_{j k}^{\mu} v_{i j k} \partial_{\mu} G_{\alpha}\left(x_{0}\right) \theta_{i}^{\alpha}+\ldots \\
& +G_{\alpha}\left(x_{0}\right) \theta_{i j k}^{\alpha} v_{i j k}+G_{\alpha \beta}\left(x_{0}\right) \theta_{i}^{\alpha} \theta_{j k l}^{\beta} v_{i j k l}+\ldots
\end{aligned}
$$

As in the proof of Theorem 2, we will construct a path along which only one coordinate $x_{i j}$ is varying at any stage, with the aim of showing that the terms linear in these coordinates are actually absent from Eq. (4.7). So, if possible, choose an initial chart and a leaf of the soul foliation along which there is dependence on some $x_{i j}$ coordinates of this chart. It is possible that the individual terms in Eq. (4.7) which depend on these coordinates are nonzero, but their sum is zero, but this can only happen for special values of $\theta_{i}$ and we are free to work within a leaf of the secondary foliation which avoids such special values. Then once again there must be dependence on the $x_{i j}$ coordinates of each chart which this leaf meets, and the proof of Theorem 2 applies to give a path along which $F$ increases without bound.

At this point we have shown that the assumed global superfield cannot depend on any of the even coordinates except $x_{0}$. Therefore in local coordinates it takes the form

$$
f(x)=f_{0}\left(x_{0}\right)+f_{\alpha}\left(x_{0}\right) \theta^{\alpha}+f_{\alpha \beta}\left(x_{0}\right) \theta^{\alpha} \theta^{\beta}+\ldots .
$$

Now, because the supermanifold is compact and nicely foliated, each leaf of the soul foliation is compact as well. Therefore, $\|f\|^{2}$, defined as in Eq. (4.1), achieves a maximum on each leaf. Then the proof of Theorem 1 applies to show that in fact there is no dependence on the odd coordinates either, and the conclusion follows.

As noted, the assumption that the supermanifolds are nicely foliated is necessary for these theorems. However, there is one important case in which it can be eliminated. 
Theorem 4. If the compact supermanifold $M$ is obtained as the quotient space of flat superspace by the action of a group $G$, then all superfields on $M$ are constant along leaves of the soul foliation.

Proof. The fact that flat superspace is covered by a single coordinate chart allows us to dispense with the technical difficulties in the previous proofs. The superfields on $M$ are just the $G$-invariant superfields on flat superspace. If such a superfield has any dependence on soul coordinates, then this dependence is polynomial and consequently some components of the superfield must be unbounded in some directions approaching infinity. This contradicts the compactness of $M$.

Theorems 2-4 also hold for noncompact supermanifolds under the assumption that each leaf is compact.

As in the case of complex manifolds, there is a corollary to Theorem 3 which states that a nice compact supermanifold $M$ cannot be embedded in a $G^{\infty}$ manner in flat superspace. A $G^{\infty}$ embedding means that $M$ obtains its $G^{\infty}$ structure from that of the surrounding space; in particular, the restriction to $M$ of a $G^{\infty}$ function in the surrounding space is $G^{\infty}$ on $M$. As in the complex case, the proof simply observes that the coordinate functions $x^{\mu}$ and $\theta^{\alpha}$ on the surrounding space are $G^{\infty}$ but cannot all be constant on leaves of $M$ since the leaves are not points.

This result can be understood more intuitively in the case of supermanifolds which are defined as solutions to algebraic equations in $B_{L}^{m, n}$. For example, consider the "supersphere" in $B_{L}^{3,0}$ defined by

$$
\left(x^{1}\right)^{2}+\left(x^{2}\right)^{2}+\left(x^{3}\right)^{2}=1 .
$$

Locally, we can solve for one coordinate in terms of the others, e.g.

$$
x^{3}=\sqrt{1-\left(x^{1}\right)^{2}-\left(x^{2}\right)^{2}},
$$

and use $x^{1}$ and $x^{2}$ as local coordinates on the supersphere. The square root is defined by its expansion in powers of the souls of the coordinates. But this expansion terminates, so there are no convergence problems and solutions can be found for any values of the soul coordinates. Hence Eq. (4.10) places no restrictions on the soul coordinates, and this subset of $B_{L}^{3,0}$ is not compact. It is in fact just the Grassmann extension of the ordinary sphere. Evidently a similar argument applies to supermanifolds defined by any polynomial equations with real coefficients.

A more physical corollary is that the only compact supermanifolds which can be useful in supergravity theory are those exceptional ones which admit $G^{\infty}$ functions with dependence on the soul coordinates, if any such exist. Superfields which do not depend on the soul coordinates cannot transform under supersymmetry and cannot represent supermultiplets of ordinary fields.

\section{Topological Imposition of Constraints}

In the previous section we showed that the global topology of a supermanifold imposes strong constraints on the globally defined superfields. This remains true even in the noncompact case. Whenever noncontractible loops exist in the 
supermanifold, there is a nontrivial requirement of single-valuedness on the superfields. In this section we will show that this requirement can be used to impose physically interesting constraints on superfields. We will construct a supermanifold which has the local geometry of flat superspace, but only admits global superfields which are chiral.

In this section only, we will use two-component Weyl spinors rather than fourcomponent Majorana spinors. Accordingly, we will allow elements of the Grassmann algebra $\theta=\theta_{\Gamma} v_{\Gamma}$ to have complex components $\theta_{\Gamma}$. Recall that a superfield on flat superspace is said to be chiral if it is invariant under the infinitesimal transformations

$$
\bar{D}(\eta)(x, \theta, \bar{\theta})=(x+i \theta \sigma \bar{\eta}, \theta, \bar{\theta}-\bar{\eta}) .
$$

These transformations have one peculiar feature. Although their action on a superfield makes sense - by transforming its arguments as above - their action on the points of superspace does not, strictly speaking. This is because the transformations do not preserve the reality of $x$ or the conjugacy of $\theta$ and $\bar{\theta}$. For our purposes it will be essential to have an action on the points of superspace. To this end we complexify $x$ and treat $\theta$ and $\bar{\theta}$ as independent complex variables.

We now consider the discrete group of transformations (5.1), where $\eta$ is reinterpreted as a complex Grassmann integer: $\eta_{\Gamma}=m+$ in for integer $m, n$. Consider the quotient space of flat superspace - which we are modeling as complexified $B_{L}^{4,4}$ - by this discrete group. Only those superfields which are invariant under (5.1) are defined on the quotient space, and as usual these are just the $G^{\infty}$ functions of the invariant variables $\theta$ and $y \equiv x+i \theta \sigma \bar{\theta}$. What has happened is that each surface of constant $y$ and $\theta$ in the flat superspace has become compactified in the quotient space so that superfields are constant along it. The quotient space itself is noncompact, however, so that many nontrivial superfields still exist.

This simple example shows that physically interesting constraints can be imposed on superfields on a supermanifold by manipulating its topology. The construction also goes through for the infinite-dimensional Grassmann algebra $B_{\infty}$ which is appropriate for physical applications. Thus, there may be applications of this mechanism in supergravity.

\section{Conclusions}

In this paper we have exhibited some of the simpler topological properties of supermanifolds, mainly those which follow from the foliation structure. We hope that we have convinced the reader that it is both possible and interesting to study the global structure of supermanifolds. Compared to the extremely rich theory of complex manifolds, virtually nothing is known about supermanifolds, and our results could be extended and generalized in many directions. The foliation structure should yield many more constraints on possible supermanifolds than those we have discussed. Generalizing our results in Sect. 4, there should be constraints on $G^{\infty}$ differential forms on compact supermanifolds. We have not addressed the question of what additional structure beyond the existence of 
foliations of the correct dimensions is needed to guarantee that a given real manifold is a supermanifold. More examples of supermanifolds are needed to suggests answers to these questions. In particular, are there supermanifolds other than simple quotient spaces which are not nicely foliated?

We believe that the extraordinarily rich structure of a supermanifold, particularly the very rigid network of foliations, should allow a complete classification. All known examples of supermanifolds are consistent with the conjecture that a maximal simply connected supermanifold is necessarily a vector bundle over its body. The qualifier "maximal" is intended to eliminate supermanifolds which are either open subsets of larger supermanifolds, or covering spaces of such subsets, since we know counterexamples in such cases. We plan to define this term precisely, and present further results supporting our conjecture, in a subsequent paper.

From the viewpoint of physical applications, certain issues are more pressing than others. First, we have restricted ourselves to supermanifolds over finitedimensional Grassmann algebras $B_{L}$, while applications to supersymmetry normally assume that an unlimited pool of independent Grassmann numbers is available. Although some of our results, such as the existence of foliations, will hold in the infinite-dimensional case, others will become nearly vacuous because compact infinite-dimensional manifolds are rare. The theory needs to be generalized in this direction. Second, we have not considered the constraints on supermanifolds which may result from the existence of a $G^{\infty}$ metric and local supersymmetry transformations. Finally, the development of an integration theory on supermanifolds is especially important [15]. Since a supermanifold is also a real manifold, the usual notion of integration does exist, but its connection with superspace integration à la Berezin is unclear [16].

After this work was completed, we learned that some of our results have also been obtained by Boyer and Gitler [17].

\section{Appendix}

The purpose of this Appendix is to describe a 3-manifold whose topology is like that of the quotient space of $B_{L}^{m, n}$ by the DSUSY group discussed in Sect. 2. This 3-manifold is similar to Rogers' example of a supermanifold with nonabelian first homotopy group [3], but in addition has torsion in its homology.

Begin with the four dimensional space $B_{2}^{1,1}$, and define the DSUSY group

$$
\begin{gathered}
T(a)(x, \theta)=(x+a, \theta), \\
Q(\eta)(x, \theta)=(x-i \eta \theta, \theta+\eta),
\end{gathered}
$$

where $a$ and $\eta$ are even and odd Grassmann numbers with integer components. We will be interested in the quotient space by this group action. Any element of this group may be written as a product of the four generators

$$
T_{0}=T(1), \quad T_{12}=T\left(v_{12}\right), \quad Q_{1}=Q\left(v_{1}\right), \quad Q_{2}=Q\left(v_{2}\right) .
$$

If we write

$$
(x, \theta)=\left(x_{0}+i x_{12} v_{1} v_{2}, \theta_{1} v_{1}+\theta_{2} v_{2}\right),
$$


then the action of the generators is

$$
\begin{gathered}
T_{0}\left(x_{0}, x_{12}, \theta_{1}, \theta_{2}\right)=\left(x_{0}+1, x_{12}, \theta_{1}, \theta_{2}\right), \\
T_{12}\left(x_{0}, x_{12}, \theta_{1}, \theta_{2}\right)=\left(x_{0}, x_{12}+1, \theta_{1}, \theta_{2}\right), \\
Q_{1}\left(x_{0}, x_{12}, \theta_{1}, \theta_{2}\right)=\left(x_{0}, x_{12}-\theta_{2}, \theta_{1}+1, \theta_{2}\right), \\
Q_{2}\left(x_{0}, x_{12}, \theta_{1}, \theta_{2}\right)=\left(x_{0}, x_{12}+\theta_{1}, \theta_{1}, \theta_{2}+1\right) .
\end{gathered}
$$

Now, taking the quotient space by the action of the subgroup generated by $T_{0}$ simply compactifies the $x_{0}$ direction into a circle without affecting the other directions at all. Hence we will consider only the three dimensional fibers over a given point on this circle, omitting reference to $x_{0}$ and $T_{0}$ from now on. We drop the subscripts on $x_{12}$ and $T_{12}$ and relabel $\theta_{1}, \theta_{2}$ as $y, z$. At this point we have a discrete group whose generators act on $R^{3}$ by

$$
\begin{gathered}
T(x, y, z)=(x+1, y, z), \\
Q_{1}(x, y, z)=(x-z, y+1, z), \\
Q_{2}(x, y, z)=(x+y, y, z+1),
\end{gathered}
$$

from which one can check the SUSY-like relations

$$
\begin{gathered}
T Q_{i}=Q_{i} T, \\
Q_{2}^{-1} Q_{1}^{-1} Q_{2} Q_{1}=T^{2} .
\end{gathered}
$$
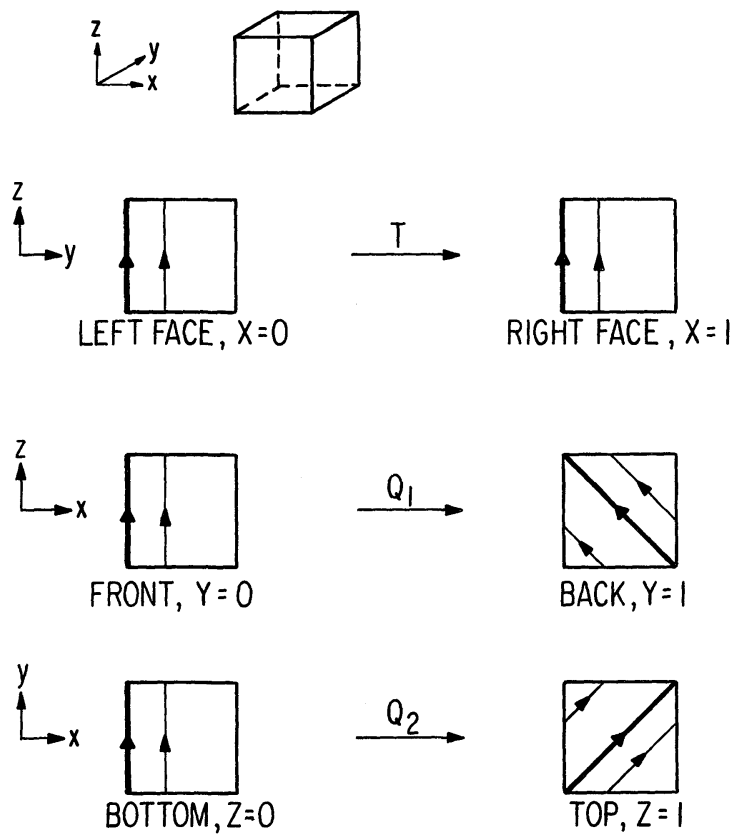

Fig. 1. The 3-manifold discussed in the Appendix is obtained from the unit cube by making the indicated identifications of opposite faces. The figures show the images of two vertical lines on each face, one being an edge, under the identification maps. Note that after the identification specified by $T$, the remaining faces are actually cylinders 
We would now like to understand the nature of the quotient space of $R^{3}$ by this group action. First, note that any point in $R^{3}$ can be brought into the unit cube $0 \leqq x, y, z<1$ in a unique way by a group transformation. Simply apply $Q_{1}$ (or its inverse) until $y$ is in the proper range, then $Q_{2}$ until $z$ is, and finally adjust the value of $x$ with $T$. Thus, the quotient space is compact and can be obtained from the cube by making identifications on the boundary.

The transformation $T$ identifies the left $(x=0)$ and $\operatorname{right}(x=1)$ faces of the cube "straight across"; i.e., in the standard manner of periodic boundary conditions. However, the identifications of front to back $\left(Q_{1}\right)$ and bottom to top $\left(Q_{2}\right)$ are twisted as shown in Fig. 1.

Any path in $R^{3}$ which joins a point to its image under a group transformation will project down to the quotient space as a closed loop which is not contractible to a point. Thus, the first homotopy group of the quotient space is generated by three loops which may be denoted $T, Q_{1}, Q_{2}$, and which satisfy the relations (A.6). Figure 2 illustrates the embedded surfaces on which the generating loops lie in the quotient space, and accounts for the fact that $T^{2}$ bounds but is not contractible whereas $T$ does not even bound. Because $T^{2} Q_{1}^{-1} Q_{2}^{-1} Q_{1} Q_{2}$ is contractible, it

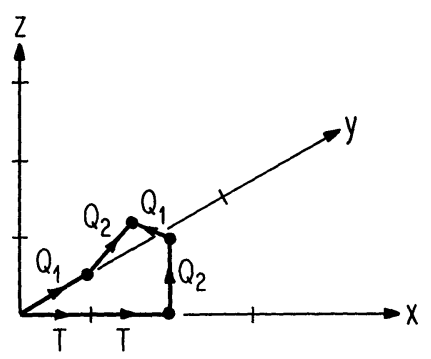

(a)
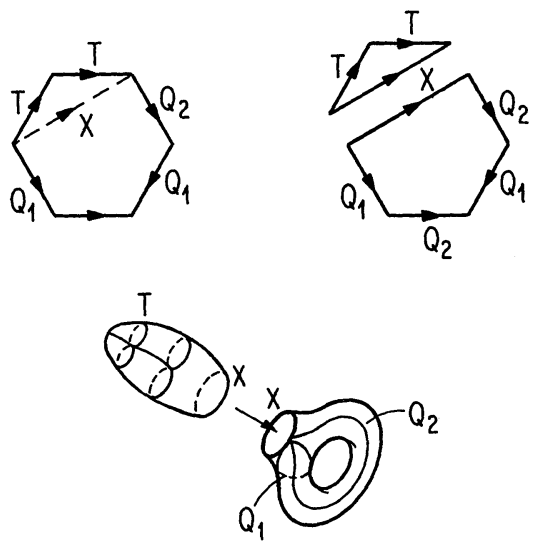

(c)

Fig. 2. a $\mathrm{A}$ loop in the covering space $\mathrm{R}^{3}$ representing $T^{2} Q_{1}^{-1} Q_{2}^{-1} Q_{1} Q_{2}$. Since this loop is contractible, it bounds a surface with the topology of a disk. $b$ A flattened version of the loop and the disk which it bounds. When this disk is projected down to the quotient space, boundary edges having the same label are identified. Cutting the disk along the edge labelled $X$ makes its image in the quotient space easier to visualize. $c$ The images of the pieces of the disk in the quotient space: $a$ crosscap and a punctured torus, joined at their boundaries $X$ 
bounds a disk in $R^{3}$ whose image in the quotient space is a crosscap joined at the boundary circle to a punctured torus. $T^{2}$ is homotopic to the boundary circle $X$, as shown. (A crosscap is a Möbius band twisted so that the single edge appears as a circle. This cannot be done in $R^{3}$ without self-intersection.)

Acknowledgements. We have enjoyed helpful discussions with P. Freund, R. Geroch, S. MacLane, R. Narasimhan, M. Rothenberg, and J. Wood.

\section{References}

1. Gates, S.J., Grisaru, M.T., Rocek, M., Siegel, W.: Superspace, or one thousand and one lessons in supersymmetry. Massachusetts: Benjamin/Cummings 1983

2. Rogers, A.: A global theory of supermanifolds. J. Math. Phys. 21, 1352-1365 (1980)

3. Rogers, A.: Some examples of compact supermanifolds with non-abelian fundamental group. J. Math. Phys. 22, 443-444 (1981)

4. Rogers, A.: Super Lie groups: global topology and local structure. J. Math. Phys. 22, 939-945 (1981)

5. Hoyos, J., Quirós, M., Ramírez Mittelbrunn, J., de Urríes, F.J.: Generalized supermanifolds. I-III. J. Math. Phys. 25, 833-854 (1984)

6. Bonora, L., Pasti, P., Tonin, M.: Supermanifolds and BRS transformations. J. Math. Phys. 23, 839-845 (1982)

7. Steenrod, N.: The topology of fiber bundles, p. 53. New Jersey: Princeton University Press 1951

8. Adams, J.F.: Vector fields on spheres. Ann. Math. 75, 603-632 (1962)

9. Abraham, R., Marsden, J.E.: Foundations of mechanics, p. 266. 2nd ed. Massachusetts: Benjamin/Cummings 1978

10. Greenberg, M.J., Harper, J.R.: Algebraic topology, a first course. Massachusetts: Benjamin/Cummings 1981

11. Kostelecký, V.A., Rabin, J.M.: A superspace approach to lattice supersymmetry. J. Math. Phys. 25, 2744-2748 (1984)

12. Bott, R., Tu, L.W.: Differential forms in algebraic topology, p. 122. Berlin, Heidelberg, New York: Springer 1982

13. Bott, R.: Lectures on characteristic classes and foliations. In: Lectures on algebraic and differential topology. Lecture Notes in Mathematics. Vol. 279, Dold, A., Eckmann, B. eds. Berlin, Heidelberg, New York: Springer 1972

Thomas, E.: Vector fields on manifolds. Bull. Am. Math. Soc. 75, 643-683 (1969)

14. Milnor, J.W., Stasheff, J.D.: Characteristic classes, p. 177. New Jersey: Princeton University Press 1974

15. Rogers, A.: Integration on supermanifolds. In: Mathematical aspects of superspace. Seifert, H.-J., Clarke, C.J.S., Rosenblum, A., eds. Dordrecht, Boston, Lancaster: Reidel 1984 Rogers, A.: Consistent superspace integration. J. Math. Phys. 26, 385-392 (1985)

16. Rabin, J.M.: The Berezin integral as a contour integral. In: Supersymmetry in physics. Kostelecký, V.A., Campbell, D.K., eds. Amsterdam, Oxford, New York, Tokyo: NorthHolland 1985. Also published as Physica 150, 65-70 (1985)

17. Boyer, C.P., Gitler, S.: The theory of $G^{\infty}$-supermanifolds. Trans. Am. Math. Soc. 285, 241-267 (1984)

Communicated by S.-T. Yau

Received November 26, 1984 